\title{
Research on Measurement of Mass, Centroid and Eccentricity of Large-scall Axisymmetric Body
}

\author{
Gang LU \\ Department of technology of \\ measurement and instrument, School \\ of Mechanical Engineering, Nanjing \\ University of Science \& Technology, \\ Nanjing, China
}

\author{
Aijun ZHANG \\ Department of technology of \\ measurement and instrument, School \\ of Mechanical Engineering, Nanjing \\ University of Science \& Technology, \\ Nanjing, China \\ Email:zaj23@163.com
}

\author{
Changming WANG \\ Department of technology of \\ measurement and instrument, School \\ of Mechanical Engineering, Nanjing \\ University of Science \& Technology, \\ Nanjing, China
}

\begin{abstract}
Pointed at low-precision of measurement of mass and centroid of large-scall axisymmetric body, measurement method is researched. After comparing, three-point method is adopted to design this system, and error is analyzed and compensated. According to experiments, measurement system has high-precision, and it can achieve requirement of engineering.
\end{abstract}

Keyword-Axisymmetric body, Mass, Centroid, Measurement, Three-point method

\section{Instruction}

In aerospace industry, we need to measure mass, centroid and eccentricity of large-scall axisymmetric body such as artificial satellite and spaceship in order to ensure that product accords with design requirements [1]. At present there are many kinds of method to measure centroid, such as suspension method, reaction force method, unbalanced moment method, multi-pivot weighing method, rotational balance method, inertia method and so on. Eccentricity is a parameter of axisymmetric body, and it is an application of measurement method for centroid. Eccentricity of axisymmetric body can be measured by multi-pivot weighing method, rotational balance method, and inertia method. Suspension method [2] can be used to measure centroid of regular and irregular body, but just small-scall object. Reaction force method can be used in measurement of large-scall object, such as vehicle. Unbalanced moment method is a method of static measurement, and it only can be used by regular small-scall object. Multi-pivot weighing method relies on static equilibrium principle, and it can be used to measure eccentricity distance of axisymmetric body with high-precision. Rotational balance method and inertia method are both dynamic measurement methods, and they are mainly used in eccentricity measurement of axisymmetric body. This system designs a suit of measurement system to measure mass, centroid and eccentricity by multi-pivot weighing method.

\section{Measurement Theory of Mass, Centroid and Eccentricity}

Measurement system adopts three-point method to measure mass, centroid and eccentricity of projectiles and rockets. Axisymmetric body is placed on a pedestal, which is supported by three sensors. According to moment equilibrium principle, mass, centroid and eccentricity can be measured at one time. Rectangular space coordinate system is built in Fig.1.
Coordinate values of three sensors' supporting-pivot are $S_{1}\left(x_{1}, y_{1}, 0\right), \quad S_{2}\left(x_{2}, y_{2}, 0\right)$ and $S_{3}\left(x_{3}, y_{3}, 0\right) \cdot$ X-axis of coordinate system passes through sensor's supporting-pivot $S_{3}$, which means $y_{3}=0, y$-axis passes through center line of pedestal, and $\left|x_{1}\right|=\left|x_{2}\right|=\left|x_{3}\right| / 2$. Disposing sensors by this means is to make sure that pressure of each sensor is pretty much the same.

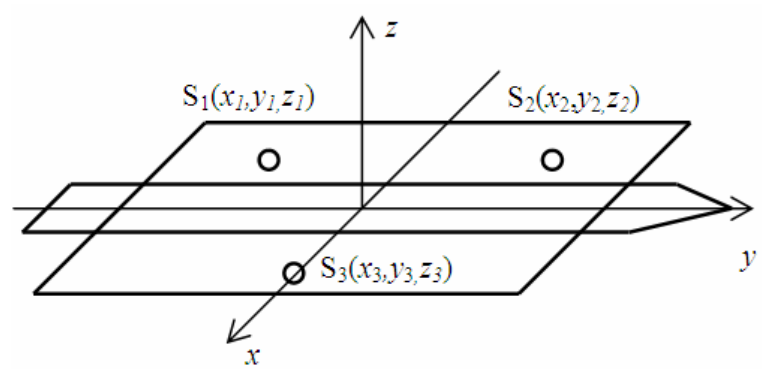

Fig. 1 Position of sensors

A Measurement theory of centroid in the axial direction.

Centroid in the axial direction $L$ is the distance between centroid of axisymmetric body and bottom of axisymmetric body. Projection of measurement device in plane oyz is given in Fig. 2.

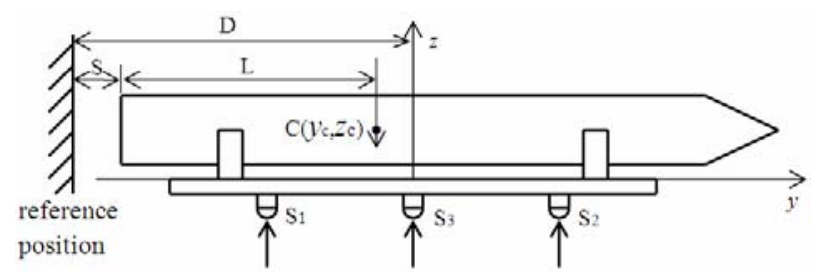

Fig. 2 Diagrammatic sketch of measurement for centroid

According to moment equilibrium principle, there is

$$
M \cdot g \times y_{c}=m_{1} \cdot g \times y_{1}+m_{2} \cdot g \times y_{2}+m_{3} \cdot g \times y_{3}
$$

$\mathrm{X}$-axis of coordinate system passes through sensor's 
supporting-pivot, so there is $y_{3}=0$.

$$
y_{c}=\frac{m_{1} \cdot g \times y_{1}+m_{2} \cdot g \times y_{2}}{M}
$$

Centroid in the axial direction of standard object is known, and the distance between reference position and bottom of standard object can be measured by grating ruler, so coordinate value of $\mathrm{x}$-axis can be calculated by Eq.2. So we can obtain calibration value of $D$ by next equation.

$$
D=L_{s}+S_{s}-y_{c}
$$

Where $D$ is calibration value; $L_{s}$ is centroid in the axial direction; $S_{s}$ is the distance between reference position and bottom of standard object.

\section{B Measurement Theory of Eccentricity}

Eccentricity of axisymmetric body includes eccentric distance and eccentric angle. Projection of measurement device in plane $O X Z$ is given in Fig. 3. We need to get weighing results in initial position and posi tion after moving around axis of axisymmetric body for $90^{\circ}$ to get eccentricity. If we can measure $\mathrm{x}$-axis coordinate value of initial position $x_{c}^{0^{\circ}}$ and $\mathrm{x}$-axis coordinate value of position after moving around for $90^{\circ} x_{c}^{90^{\circ}}$, we will get eccentric distance and eccentric angle. According to moment equilibrium principle:

$$
x_{c}=\frac{m_{1} \times x_{1}+m_{2} \times x_{2}+m_{3} \times x_{3}}{M}
$$

$x_{c}^{0^{\circ}}$ and $x_{c}^{90^{\circ}}$ can be measured according to equation (4), so eccentric distance and eccentric angle can be calculated by Eq.5 and Eq.6.

$$
e=\sqrt{\left(x_{c}^{0^{\circ}}\right)^{2}+\left(x_{c}^{90^{\circ}}\right)^{2}}, \quad \theta=\arctan \left(\frac{x_{c}^{90^{\circ}}}{x_{c}^{0^{\circ}}}\right)
$$

Where $e$ is eccentric distance and $\theta$ is eccentric angle.

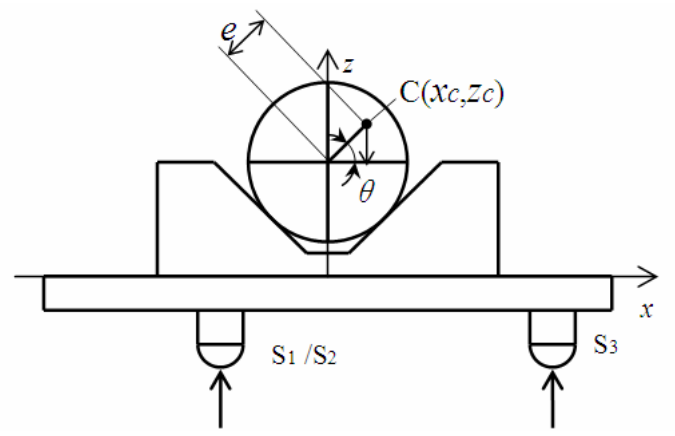

Fig. 3 Diagrammatic sketch of measurement for eccentricity

\section{Error Analysis and Compensation}

From measurement theory of mass, centroid and eccentricity we can see that mass error mainly comes from measurement error of sensors; centroid error mainly comes from measurement error of sensors, position error between each sensor and measurement error of grating ruler; eccentricity error mainly comes from measurement error of sensors, position error between each sensor and angle error brought from rotating axisymmetric body. In this paper, we only analyze eccentricity error. According to the measurement theory of eccentricity, eccentric distance and eccentric angle can be calculated by $X_{c}$ of initial position and position after rotating for $90^{\circ}$. In our measurement system, we measure $x_{c}$ of initial position, position after rotating for $90^{\circ}$, $180^{\circ}$ and $270^{\circ}$ to compensate error. We compensate error of mechanical structure by measurement of symmetrical position in order to minish error. From equation (4) error of $x_{c}$ brought by measurement error of sensor is as follows:

$$
\begin{aligned}
\sigma_{x_{C}}=\sqrt{\left(\frac{\partial x_{C}}{\partial m_{1}^{2}} \sigma_{1}\right)^{2}+\left(\frac{\partial x_{C}}{\partial m_{2}^{2}} \sigma_{2}\right)^{2}+\left(\frac{\partial x_{C}}{\partial m_{3}^{2}} \sigma_{3}\right)^{2}} \\
\frac{\partial x_{c}}{\partial m_{1}^{2}} \sigma_{1}=m_{2}\left(x_{1}-x_{2}\right)+m_{3}\left(x_{1}-x_{3}\right) \\
\frac{\partial x_{c}}{\partial m_{2}^{2}} \sigma_{2}=m_{1}\left(x_{2}-x_{1}\right)+m_{3}\left(x_{2}-x_{3}\right) \\
\frac{\partial x_{c}}{\partial m_{3}^{2}} \sigma_{3}=m_{1}\left(x_{3}-x_{1}\right)+m_{2}\left(x_{3}-x_{2}\right)
\end{aligned}
$$

Where $x_{1}=-50, x_{2}=-50, x_{3}=100$.

When we test standard object whose mass is $39269.7 \mathrm{~g}$ and centroid is $320 \mathrm{~mm}$, error of $x_{c}$ brought by measurement error of sensors is as follows:

$$
\sigma_{x_{c}^{0^{\circ}}} \approx \sigma_{x_{c}^{900^{\circ}}} \approx \sigma_{\chi_{c}^{180^{\circ}}} \approx \sigma_{x_{c}^{277^{\circ}}} \approx 0.000066 \%
$$

So eccentricity error is $\sigma_{e}=0.000047 \%$. We can find that eccentricity error brought by measurement error is very small.

\section{System Testing}

After sensors calibration, we use standard object to calibrate mass, centroid and reference position of grating ruler of eccentricity measurement device. Standard object's length is $640 \mathrm{~mm}$, position of centroid is $320 \mathrm{~mm}$ and mass is $39269.7 \mathrm{~g}$. Data and result of calibration is in Table 1. 
Table 1 Data and result of calibration of mass, centroid and eccentricity

\begin{tabular}{llccc}
\hline & Sensor 1 & Sensor 2 & Sensor 3 & Total mass \\
\hline Mass [g] & 12584.08 & 13935.24 & 12754.13 & 39273.4 \\
Grating ruler[mm] & & \multicolumn{2}{c}{268.88} & \\
result of calibration [mm] & & \multicolumn{2}{c}{$D=583.719$} & \\
\hline
\end{tabular}

Standard object is tested by measurement system. Measurement result is in Table 2.

Table 2 Measurement result of mass, centroid and eccentricity

\begin{tabular}{|c|c|c|c|c|}
\hline & Rotating angle & Sensor 1 & Sensor 2 & Sensor 3 \\
\hline \multirow{4}{*}{$\begin{array}{l}\text { Value of sensor in each } \\
\text { angle[g] }\end{array}$} & $0^{\circ}$ & 12584.8 & 13935.2 & 12754 \\
\hline & $90^{\circ}$ & 12611.6 & 13909.4 & 12753.4 \\
\hline & $180^{\circ}$ & 12635.2 & 13887.1 & 12751.6 \\
\hline & $270^{\circ}$ & 12654.6 & 13865 & 12752.9 \\
\hline Grating ruler[mm] & \multicolumn{4}{|c|}{268.75} \\
\hline \multirow[t]{2}{*}{ Measurement result } & Total mass[g] & Centroid[mm] & $\begin{array}{l}\text { eccentric } \\
\text { distance[mm] }\end{array}$ & $\begin{array}{l}\text { eccentric } \\
\text { angle }\left[^{\circ}\right]\end{array}$ \\
\hline & 39274 & 320.127 & 0.00466 & 1.56 \\
\hline
\end{tabular}

\section{Conclusions}

Measurement of axisymmetric body's mass character has important significance in estimating and improving its work efficiency. Measurement system introduced in this paper can measure mass, centroid and eccentricity with high-precision. According to error analysis of measurement theory and testing of final product, the whole system has perfect functional and reliable performance.

\section{References}

[1] W. Hou: Journal of North University of China (Natural Science Edition), Vol.29 (2008) No.6, p.505. (In Chinese)

[2] Danis and Norman J: IEEE Transactions on Aerospace and Electronic Systems, Vol.2 (1993), p.412.

[3] Lafond, D., Duarte, M. and Pince,F: Journal of Biomechanics, Vol.9 (2004), p.1421.

[4] G. N. Yao, J. H. Hu, X. L. Zhao and G. J. Sun: Optics and Precision Engineering, Vol.17 (2009) No.5, p.1141. (In Chinese)

[5] Y. B. Qian and B. Wu: Journal of Projectiles, Rockets, Missiles and Guidance, Vol.26 (2006) No.3, p.126. (In Chinese) 